\title{
Redefining surgery and surgical profession
}

\author{
Hans G. Beger UIm
}

Published online: 27 February 2010

(C) Springer-Verlag 2010

The present-day surgeon is the leader of a safe, highperformance team, providing evidence-based best surgical practice to his patients. The surgeon is trained to handle images; he is able to protect tissue by minimal invasive approaches, as well as by open traditional surgery. Surgeons are strongly concerned with minimizing procedure-related postoperative morbidity and hospital mortality; however, surgeons are increasingly aware that surgical success is determined by long-term outcome and patient's satisfaction with the treatment.

New competing surgical measures like minimal invasive technologies, the use of intraluminal endoscopic surgical treatment modalities as well invasive radiological and intraluminal vascular technologies have changed surgical practice in all specialities. The major benefits of the new tools are minimizing of surgical trauma and shortening of hospital stay; both are becoming standard goals for surgical treatment. As a consequence of the rapidly developing minimal surgical specialities, new outcome determinants have been defined. The major outcome determinant of surgical treatment has been and still is hospital mortality. However, for elective surgical procedures, a hospital mortality of below $5 \%$ is recommended even when major operative procedures like organ transplantation, pancreatic head resection, and esophagus resection are applied. Zero mortality has become the new lighthouse of elective surgery as demonstrated by many experienced surgeons for major surgical procedures and is recommended for all elective minimal invasive procedures as well. An increasingly important criterion of surgical success is long-term outcome which determines the real patient's benefit of surgical treatment.

H. G. Beger Ulm $(\bowtie)$

c/o University of Ulm,

Ulm, Germany

e-mail: hans.beger@uniklinik-ulm.de
The surgeon's work and the surgical institutions are under a permanent quality control to apply best surgical practice based on best clinical evidence.

Prior to doing surgery in patients, surgical training courses has become, in many surgical institutions and for surgical societies, a major goal to improve surgical skills, to standardize procedures, and to minimize surgery-related complications particularly in minimal invasive surgery. Surgical training courses are offered for young surgeons to become a qualified specialized surgeon. In this issue (Langenbeck's Arch Surg. 2010;395:DOI:10.1007/s00423-009-0568-7), J. Ch. Lauscher from Berlin presented the long-term effects about sustainability of skill courses for General and Visceral Surgery in the journals section of Training and Education in Surgery. They were able, in a large cohort of participants (459 of 756 participants in a 1-week course), to demonstrate that participants in the course have long-term profit and improvement of their surgical technique. The evaluation showed additionally that $89.5 \%$ of the participants wanted skill courses to become an integral part of surgical training. Training of young surgeons and professionally instituted education of surgical societies and institutions contribute not only to the motivation of young surgeons but also to the high level of surgical practice.

In cancer surgery, as well as in surgical treatment of inflammatory diseases, the application of best practice is closely linked to bedside transformation of research knowledge and a scientific background of decision making. Applied best clinical surgical practice in cancer surgery needs to incorporate the molecular profile of the individual cancer to apply personalized medicine. Many of the surgical treatment modalities are part of management concepts which include a variety of evident medical treatment concepts; multidisciplinary treatment has become dominant and more effective in surgery replacing surgical monotherapy. Surgical professionalism is based on participation on continued professional developments and education. A surgeons' professional life is dedicated to continuity of care and to cooperation with other colleagues and professions. 